\title{
The visual evoked response in hysterical amblyopia
}

\author{
JOAN BEHRMAN \\ Electrodiagnostic Clinic, Moorfields Eye Hospital, London
}

Hysterical amblyopia is a term used to describe a syndrome in which reduction of visual acuity accompanied by contraction of the visual fields occurs in the absence of any organic lesion. This syndrome has been the subject of many reports (Eames, I947; Yasuna, I95I ; Schlaegel and Quilala, I955; Krill, I967), but no conclusive diagnostic test has yet been established. Spiralling of the visual fields, and tubular fields which remain unaltered when examined at different distances or with objects of different size, are necessarily of subjective origin and suggestive of hysterical amblyopia (Traquair, 1949). Krill (1967) noted an upward shift in the threshold of the dark-adaptation curve after prolonged testing, which he termed the "exhaustion phenomenon" and claimed to be a unique abnormality occurring in hysteria.

The most common presenting complaint is of a decrease in vision, which may be associated with headache, night blindness, diplopia, etc.; or there may be no ocular complaints and constricted fields are found on general ophthalmic examination (Heaton, 1968). The condition is not unusual among school children (Eames, 1947).

The degree to which the symptoms of hysterical amblyopia can mimic those of organic disease is such that in many cases the psychogenic nature is difficult to establish. Electrodiagnostic tests can confirm the integrity of the retina and visual pathways and are a useful aid in differential diagnosis. A number of patients with unexplained reduction of vision have been referred to the Electrodiagnostic Clinic and the methods of analysis used and the results, which have some bearing on the nature of hysterical amblyopia, are the subject of this paper.

\section{Material}

35 patients ( 12 male and 23 female) were referred to the Electrodiagnostic Clinic at Moorfields Eye Hospital between 1965 and 1968 . Their ages ranged from 7 to 67 years, but eighteen ( 51 per cent.) were under 16 years old.

The chief presenting symptom in thirty of the 35 patients was a bilateral blurring of vision or deterioration of visual acuity or, in the case of the children, an inability to see the blackboard clearly. This was associated with headaches in five, diplopia in one, and night blindness in four patients, including one who thought her symptoms were due to avitaminosis A caused by a period in a concentration camp during the war. One patient, whose mother had retinitis pigmentosa, complained of poor side vision.

Of the remaining five, two suffered from headaches only, one had difficulty playing ball games, and in two the constricted fields were discovered during examination for non-ocular conditions.

All patients had a complete ophthalmological examination and several had neurological or psychiatric investigations in addition. 


\section{Methods}

VISUAL AGUITY

The best corrected visual acuity was recorded in all patients, either by the referring ophthalmologist or at the Clinic.

\section{VISUAL FIELDS}

These had in most cases been previously plotted by the referring ophthalmologist; the investigation was repeated at the clinic either on the Goldmann perimeter using several targets including the largest at maximum intensity, or in a few cases on the Lister perimeter using a $3^{\circ}$ white target. In several cases, central fields were also tested on a Bjerrum screen using a variety of test-object sizes and patient-screen distances.

EOG AND ERG

In all patients the electrooculogram (EOG) and the electroretinogram (ERG) were recorded. The EOG technique was identical to that described by Arden, Barrada, and Kelsey (1962). The ERG was elicited by a stimulus from a $\frac{1}{4}$ Joule Dawe strobotorch and recorded via a Karpe contact lens electrode on a Schwarzer polygraph machine, the PEE 4. Except in the very young patients, the ERG was recorded during a Io-min. period of dark adaptation.

\section{DARK ADAPTATION}

The dark-adaptation curve was plotted for each patient on the Goldmann-Weekers dark adaptometer. The test was preceded by an instruction period and an initial light adaptation to a diffuse light produced by two 6o-watt bulbs reflected from the white interior of the globe for $5 \mathrm{~min}$., after which threshold measurements were commenced.

The subject fixed a small red light, located a few degrees above the test light, which subtended i ${ }^{\circ}$ at the eye and flashed once a second. Thresholds were plotted by gradually increasing the stimulus light intensity until the flashes could just be observed by the patient. This was done every 1 or 2 min. for some 20 to $50 \mathrm{~min}$. in the dark. or until no further change in threshold occurred.

\section{VISUAL EVOKED RESPONSE}

In 22 of the patients the visual evoked response (VER) was recorded from a unipolar electrode placed on the midline $2 \mathrm{~cm}$. above the inion. The ground and indifferent electrodes were clipped to the ear lobes. Responses were elicited to flashes produced by the Dawe strobotorch at a rate of approximately 3 per sec., with one filter of red "Cinemoid" (No. 6) inserted. The responses were amplified, monitored on a Tektronix 502 oscilloscope, and averaged for 1 min. using a T.M.C. C.A.T.400 with a sweep time of $250 \mathrm{msec}$. The final result was written out on the D.C. channel of the polygraph machine. In all records, an upward deflection represents positivity at the unipolar electrode.

By this method, routine light-adapted VERs were produced. To record dark-adapted or peripheral VERs the method described by Adams, Arden, and Behrman (1969) was employed. In this procedure, a green and blue filter (Cinemoid, Nos 20 and 39) were used together with neutral density filters (Cinemoid, No. 6o) to reduce the intensity of the light from the strobotorch. A segment of a pingpong ball was placed over one eye to diffuse the light evenly over the retina. The other eye was covered and the patient was left in the dark. VERs were recorded by a method identical to that described for light-adapted VERs, except that the summation continued for a further 30 sec., i.e. for $1 \frac{1}{2}$ min. The first record was started after $30 \mathrm{sec}$. in the dark with neutral density filters equivalent to $2 \log$ units inserted. Recording continued at intervals during dark adaptation and more neutral density filters were inserted as dark adaptation progressed, so that threshold or slightly larger than 
threshold traces were recorded throughout. This was continued until a threshold response could just be evoked with filters equivalent to $4 \log$ units interposed in addition to the green and blue ones already there.

Evidence that the cortical responses evoked in this manner are related to rod activity in the eye has been obtained by plotting in a modified form the spectral sensitivity curve for these responses and by examining the dark-adapted or peripheral VERs in various known clinical conditions. VERs recorded in this way have a recognizably different waveform with a longer latency than the more usually obtained light-adapted VERs, and this is further evidence that they are derived from the rod system (Adams and others, 1969).

FOLLOW-UP STUDIES

Thirteen of the patients were followed up at intervals ranging from 6 months to 3 years.

\section{Results}

Despite age and sex differences, the patients in the series form a homogeneous group and one case history will suffice.

A ro-year-old girl gave a history of difficulty in reading the blackboard at school for the past year and more recently of headaches and dizziness. She had had recurrent otitis media when younger. There was no relevant family history; the Wassermann reaction and Kahn test were negative.

\section{Examination}

The fundi were normal apart from slight pigmentary mottling at the periphery. Visual acuity was $6 / 36$ in both eyes and N.24 with no refractive error. Visual fields showed a generalized constriction to within $20^{\circ}$ of fixation with a $3 \mathrm{~cm}$. target at $2000 \mathrm{~mm}$. She was referred to the Electrodifygnositic Clinic with a diagnosis of a retinal dystrophy.

EOG and ERG were normal, as was the light-adapted VER. A final threshold I log unit above normal was recorded on the dark-adaptation curve. The dark-adapted VER threshold was, however, within normal limits, which suggested that she was in fact suffering from hysterical amblyopia. Psychiatric interview revealed a shy, diffident girl, who unlike her brighter younger sister was having difficulty in keeping up in class. Interestingly, she also had a strong fear of the dark. When she obtained a place at a grammar school her symptoms remitted and her visual acuity improved to $6 / 6$.

\section{GLINICAL EXAMINATION}

In all cases the ocular media were clear and fundus examination revealed no abnormality. Intraocular pressure where measured was normal, and neurological investigations were negative.

\section{VISUAL ACUITY}

The best corrected visual acuity in all patients was $6 / 9$ or less. In two-thirds the visual acuity was between $6 / 18$ and $6 / 36$, and in two it was reduced to counting fingers.

\section{VISUAL FIELDS}

In 28 cases the visual fields were concentrically constricted to within $20^{\circ}$ of fixation and in nineteen cases to within $10^{\circ}$ or less. In five cases the fields were constricted to within $20^{\circ}$ 
to $30^{\circ}$; included in these are two patients whose fields had expanded since their first examination by an ophthalmologist. Two of the patients had contracted vision with spiralling fields.

EOG AND ERG

The EOG showed a normal light-induced rise in potential in every patient and the amplitude of the ERG was normal and increased normally with dark adaptation.

\section{DARK ADAPTATION}

All patients with fields constricted to within $20^{\circ}$ of fixation had abnormal final threshold sensitivities as measured on the Goldmann-Weekers dark adaptometer. In only one case could the "exhaustion phenomenon" (Krill, I967) be convincingly demonstrated.

A direct relationship was found in our series between the degree of constriction of the visual field and the elevation of the dark-adapted final threshold above normal. Fields constricted to $10^{\circ}$ or less were associated with a monophasic dark-adaptation curve, and those between $10^{\circ}$ and $20^{\circ}$ with a significantly raised final threshold. Where the fields were only moderately constricted, the mean dark-adapted final threshold was not above normal (Table, opposite).

LIGHT-ADAPTED VER

This was of normal amplitude in all cases in which it was recorded.

DARK-ADAPTED VER

The technique for recording dark-adapted VERs was developed as a result of our seeing a number of patients who had normal EOGs, ERGs, and light-adapted VERs and whose only abnormality appeared to lie in the subjective tests in which the peripheral retina was involved, i.e. visual fields and dark adaptation.

Dark-adapted VERs were therefore recorded only in the seventeen patients seen most recently. In fifteen cases the criterion established by normal subjects was reached, in that threshold or slightly larger than threshold evoked responses were recorded from the cortex after 12 to 15 minutes' dark adaptation, with neutral density filters equivalent to $4 \log$ units plus the blue-green filters interposed. A typical set of tracings recorded during dark adaptation are shown in the Figure (opposite). The normal results obtained by this method are in contrast to the abnormal subjective thresholds recorded in these patients on the dark adaptometer, which averaged $2 \cdot 8 \mathrm{log}$ units above normal (range $0 \cdot 3$ to $6 \cdot 0$ ).

In one patient the dark-adapted VER was technically unsatisfactory. In the remaining $N$ case the dark-adapted VER was barely recordable with a relatively intense stimulus, and $N$ this will be discussed.

FOLLOW-UP STUDIES

These showed that the visual acuity returned to normal and the fields became less constricted in seven of the children. (It was noted that fields measured by confrontation methods improved more quickly than those plotted on the perimeter.) Three children and two adults who were reviewed showed no significant change; one adult showed a decrease in visual acuity. 


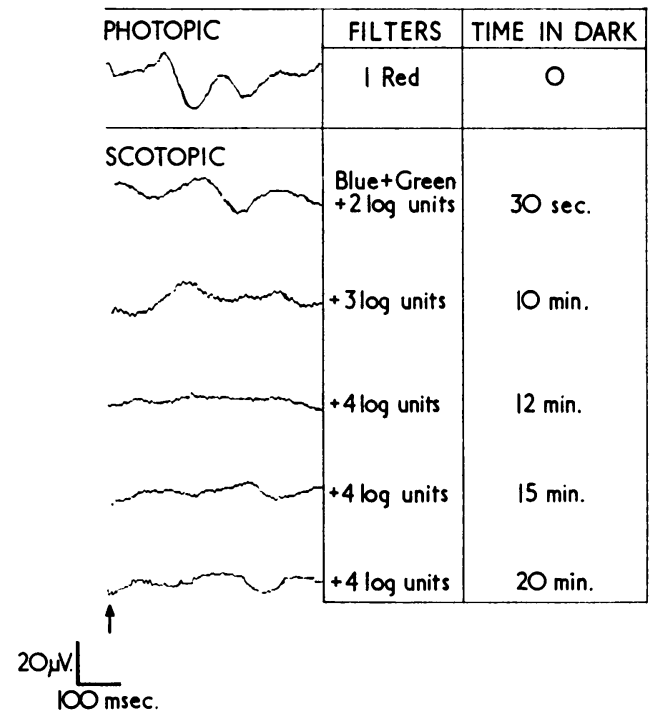

Table Relation between field size and final threshold sensitivity on dark-adaptation curve. Initial and follow-up results combined

\begin{tabular}{cl}
\hline Field size & $\begin{array}{l}\text { Dark-adaptation curve } \\
\text { Final threshold sensitivity } \\
\text { (log units) }\end{array}$ \\
\hline $0-10^{\circ}$ & $4 \cdot 4 \pm \mathrm{I} \cdot 2$ Log units \\
$11^{\circ}-20^{\circ}$ & $3 \cdot 4 \pm \mathrm{I} \cdot 7$ \\
$21^{\circ}-30^{\circ}$ & $1 \cdot 4 \pm 0.6$ \\
Normal & $1 \cdot 0 \pm 0.5$ \\
\hline
\end{tabular}

FIG. I Photopic and scotopic VERs in a patient with hysterical amblyopia.

The photopic response was recorded under conditions of light adaptation with one red filter.

The scotopic responses were recorded serially during dark adaptation with increasing neutral density filters as dark adaptation progressed. Thus after 12 minutes in the dark, the stimulus light was below threshold with filters of 4 log units; after 20 minutes' dark-adaptation the same stimulus evoked a good VER.

This should be compared with the threshold reached on the subjective dark-adaptation curve, which remained at $2.5 \log$ units above normal after 30 minutes in the dark.

\section{Discussion}

Most organic disorders of the eye causing visual symptoms are readily detected by normal clinical methods. Electrodiagnostic techniques are valuable in elucidating the cause of visual dysfunction in cases in which the retina appears to be normal. Riggs (1954) showed that the electroretinogram was of use in differentiating the various forms of night blindness. Both the ERG and the EOG may demonstrate abnormalities of retinal function in the presence of a clinically normal fundus, particularly in the early stages of a tapeto-retinal degeneration.

The patients in our series exhibit many features similar to those described in previous series:

(x) Reduced visual acuity as the chief presenting complaint in the majority of cases.

(2) Concentric contraction of the visual fields with constant size on repeated plotting (Yasuna, 195 I; Schlaegel and Quilala, 1955).

(3) Abnormal dark-adaptation curve (Krill, 1967).

(4) A high proportion of children and females in the series (Yasuna, 195 I Schlaegel and Quilala, 1955; Krill, 1967).

(5) Occasional remission of symptoms (Eames, 1947; Friesen and Mann, 1966).

We may therefore conclude that our patients also are suffering from hysterical amblyopia.

Apart from four adults in the series with a known psychiatric history and one of the children who had in the past suffered from a hysterical paralysis of the arm, psychiatric assessment, when carried out, revealed only that the onset of symptoms tended to coincide with a period of stress. This was particularly true in some of the children who were 
studying for examinations, after which the symptoms remitted. This remission of symptoms is evidence in favour of a psychogenic condition, as most organic disorders producing contracted fields are either progressive or irreversible.

The finding not only that the final threshold of the dark-adaptation curve was above normal, but also that the degree of abnormality was related to the degree of constriction of the visual fields, indicated a generalized consistency of responses not usually associated with hysterical conversion syndromes. Moreover, the fact that we were unable to demonstrate Krill's "exhaustion phenomenon" in most cases made it desirable for us to evolve further methods of investigation.

This led to the development of the dark-adapted VER technique, in which successive VERs are recorded in an objective assessment of dark adaptation. In the majority of patients, normal results were obtained, proving that the suppression of vision occurred after the specific cortical response had been evoked. This is, at present, the best definition of a psychogenic disturbance that one can obtain. The fact that in one case a darkadapted VER could be detected only at higher stimulus intensities could perhaps be due to the known great individual variation in the size of the VER. Alternatively, it could be the result of a more widespread inhibitory process. In one other, with a unilateral psychogenic visual disturbance, abnormal dark-adapted VERs were recorded from the affected eye (Adams and others, 1969).

These findings in the VERs in hysterical amblyopia are similar to those obtained in studies of the cortical evoked activity in other modalities. Alajouanine, Scherrer, Barbizet, Calvet, and Verley (1958) stimulated the ulnar nerve in seven patients suffering from unilateral hysterical anaesthesia. The somato-sensory evoked potentials were identical from the two sides. However, Hernández-Peón, Ghávez-Ibarra, and Aguilar-Figueroa ( 1963 ) noted, in one similar case, that the somato-sensory evoked responses were reduced when the affected arm was stimulated, but that the amplitude increased to normal under barbiturate anaesthesia or following a painful stimulus.

It is interesting that hypnosis has been found to have no effect on the VER. Similar VERs were recorded before hypnosis and under hypnosis in which it was suggested that alterations would occur in the brightness and size of a fixed stimulus (Beck and Barolin, 1965). The changes produced subjectively by the hypnosis would seem therefore to occur at a level beyond the primary receptor cortex. In the majority of cases in our series, in which the peripheral VERs were normal, the inhibition of vision appears to occur at a similar stage, whereas in the one bilateral and one unilateral case mentioned, there appears to be a more generalized suppression which does affect the VER.

\section{Summary}

The syndrome in which reduced visual acuity is the main presenting symptom and constricted fields and poor dark adaptation are found on examination is known as hysterical amblyopia. It is not uncommon and 35 cases are reported here. In each case electrodiagnostic tests, including the light-adapted VER, supported the clinical finding that there was no organic lesion.

The elevation of the final threshold of the dark-adaptation curve was shown to be related to the degree of constriction of the visual fields. Therefore, a technique for recording the dark-adapted VER was developed to provide an objective test of peripheral retinal function. A normal dark-adapted VER, recorded despite a poor subjective darkadaptation curve, is at present the best indication of hysterical amblyopia. 
I should like to thank Dr. G. B. Arden, who supervised this study, and Mr. J. H. Kelsey and Dr. R. Levy for their help and advice.

\section{References}

AdAMS, L., ARDEN, G. B., and Behrman, J. (1969) Brit. J. Ophthal., 53, 439

ALAJOUANINe, T., SGHERRER, J., BARBIZET, J., CALVET, J., and VERLEY, R. (1958) Rev. neurol., 98, 757 ARDEN, G. B., BARRADA, A., and KELSEY, J. H. (1962) Brit. F. Ophthal., 46, 449

BECK, E. C., and BAROLIN, G. s. (1965) F. nerv. ment. Dis., I40, I 54

eames, т. H. (1947) Amer. F. Ophthal., 30, 610

FRIESEN, H., and MANN, w. A. (1966) Amer. F. Ophthal., 62, i 106

heaton, J. M. (1968) "The Eye: Phenomenology and Psychology of Function and Disorder".

Tavistock Publications, London

HERNÁNDEZ-PEÓN, R., CHÁVEZ-ibARRA, G., and AGUilar-figueroA, E. (1963) Electroenceph. clin.

Neurophysiol., 15, 889

KRILL, A. E. (1967) Amer. 7. Ophthal., 63, 230

RIGGS, L. A. (1954) Ibid., 38, no. I, pt 2, p. 70

SGHLAegel, T. F., and Quilala, F. v. (1955) A.M.A. Arch. Ophthal., 54, 875

TRAQUAIR; H. M. (1949) “An Introduction to Clinical Perimetry", 6th ed. Kimpton, London yasuna, E. R. (195I) A.M.A. Arch. Ophthal., 45, 70 